OPEN ACCESS

Edited by: Cesare Piazza,

University of Brescia, Italy

Reviewed by:

Renata Ferrarotto,

University of Texas MD Anderson

Cancer Center, United States

Christophe Le Tourneau, Maria Sklodowska-Curie National

Research Institute of Oncology,

Poland

${ }^{*}$ Correspondence:

Alhadi Almangush

Alhadi.almangush@helsinki.fi

Antti A. Mäkitie

antti.makitie@helsinki.fi

Specialty section:

This article was submitted to

Head and Neck Cancer,

a section of the journal

Frontiers in Oncology

Received: 12 October 2020

Accepted: 29 January 2021

Published: 08 March 2021

Citation:

Almangush A, Leivo / and Mäkitie AA

(2021) Biomarkers for Immunotherapy

of Oral Squamous Cell Carcinoma:

Current Status and Challenges.

Front. Oncol. 11:616629.

doi: 10.3389/fonc.2021.616629

\section{Biomarkers for Immunotherapy of Oral Squamous Cell Carcinoma: Current Status and Challenges}

\author{
Alhadi Almangush ${ }^{1,2,3,4,5 *}$, Ilmo Leivo ${ }^{4,6}$ and Antti A. Mäkitie $2,7,8^{*}$ \\ ${ }^{1}$ Department of Pathology, University of Helsinki, Helsinki, Finland, ${ }^{2}$ Research Program in Systems Oncology, Faculty of \\ Medicine, University of Helsinki, Helsinki, Finland, ${ }^{3}$ Department of Oral and Maxillofacial Diseases, University of Helsinki, \\ Helsinki, Finland, ${ }^{4}$ Institute of Biomedicine, Pathology, University of Turku, Turku, Finland, ${ }^{5}$ Faculty of Dentistry, University of \\ Misurata, Misurata, Libya, ${ }^{6}$ Department of Pathology, Turku University Central Hospital, Turku, Finland, ${ }^{7}$ Department of \\ Otorhinolaryngology - Head and Neck Surgery, University of Helsinki and Helsinki University Hospital, Helsinki, Finland, \\ ${ }^{8}$ Division of Ear, Nose and Throat Diseases, Department of Clinical Sciences, Intervention and Technology, Karolinska \\ Institutet and Karolinska University Hospital, Stockholm, Sweden
}

Oral squamous cell carcinoma (OSCC) forms a major health problem in many countries. For several decades the management of OSCC consisted of surgery with or without radiotherapy or chemoradiotherapy. Aiming to increase survival rate, recent research has underlined the significance of harnessing the immune response in treatment of many cancers. The promising finding of checkpoint inhibitors as a weapon for targeting metastatic melanoma was a key event in the development of immunotherapy. Furthermore, clinical trials have recently proven inhibitor of PD-1 for treatment of recurrent/metastatic head and neck cancer. However, some challenges (including patient selection) are presented in the era of immunotherapy. In this mini-review we discuss the emergence of immunotherapy for OSCC and the recently introduced biomarkers of this therapeutic strategy. Immune biomarkers and their prognostic perspectives for selecting patients who may benefit from immunotherapy are addressed. In addition, possible use of such biomarkers to assess the response to this new treatment modality of OSCC will also be discussed.

Keywords: oral squamous cell carcinoma, immunotherapy, biomarkers, immune response, survival

\section{INTRODUCTION}

Survival rate of oral squamous cell carcinoma (OSCC) is about $50 \%$ of affected cases. Advances in traditional treatments (surgery, radiotherapy, chemotherapy) of OSCC have failed to increase survival and, at the same time, they have been associated with significant side effects. Prediction of survival in oral cancer depends on classical parameters such as tumor grade and depth of invasion, although many biomarkers have been introduced as potential prognosticators of OSCC $(1,2)$.

Recent research has introduced immunotherapy as an effective treatment option for OSCC. The hypothesis of immunotherapy was based on a theory that was introduced for more than a century ago postulating an ability of the immune system to repress cancer cells and aid in patient recovery (3). The significance of cancer immunotherapy was recognized more universally when the Nobel Prize in 
Physiology or Medicine was awarded for the development of such therapies in 2018 (4). For OSCC, immunotherapy was firstly approved for recurrent/metastatic cases (similar to other cancers of head and neck region) (5). Of note, neoadjuvant immunotherapy administered preoperatively has been recently introduced for untreated OSCC (6).

With the success of immunotherapy in the treatment of OSCC, it has become important to find parameters to select patients who might benefit from this treatment strategy as well as to find a predictive marker/s for following treatment response. In this minireview we will discuss different methods that have been introduced to assess the immune response and immune biomarkers in OSCC.

\section{ASSESSMENT OF IMMUNE RESPONSE AS A PART OF GRADING SYSTEMS OF OSCC}

Immune cells are among the main cellular components of cancer stroma tissue (7). The interaction of immune cells with tumor cells has been widely studied as one of the factors that influence tumor progression $(8,9)$. It has been reported in many cancers that active antitumor immune response is a feature of good prognosis $(9,10)$. Many proposals have suggested to assess the immune response as a part of histopathologic grading of OSCC. For example, an early study by Anneroth et al. (11) suggested to incorporate the assessment of the inflammatory cell infiltrate as a part of their malignancy grading system (11). They scored lymphoplasmacytic infiltrates into four categories as marked, moderate, slight or none (11). That system was modified later by Bryne et al. (12) who assessed malignancy grade (including the lymphoplasmacytic infiltrate) at the invasive front of OSCC (12). Brandwein-Gensler et al. (13) assessed the immune response as a part of a histologic risk score including three parameters: worst pattern of invasion, perineural invasion and lymphocytic host response (13). Most recently (2020), Bjerkli et al. proposed a histo-score based on the assessment of the lymphocytic infiltrate and tumor differentiation, and showed that the score gave a good prediction of survival in oral tongue cancer (14). Our group (15) proposed stromal classification, based on the assessment of tumorinfiltrating lymphocytes and tumor-stroma ratio, with a promising prognostic value in early oral tongue cancer.

From the above historically accumulated evidence, it seems that incorporation of the immune response as a part of the grading system of OSCC is a useful and important step which has not yet been implemented in pathology practice. A clinically relevant grading system with a robust association with tumor behavior and outcome, which considers the immune response is expected to become very useful for future immunotherapy of OSCC.

\section{HISTOLOGIC SEMIQUANTITATIVE ASSESSMENT OF TUMOR-INFILTRATING LYMPHOCYTES}

Morphological evaluation of tumor-infiltrating lymphocytes (TILs), using routine hematoxylin eosin (HE)-stained tumor sections, has been reported in many cancers including OSCC (16). A standardized method for the assessment of TILs has been introduced by the International TILs Working Group (9). Accumulating evidence has shown the significance of this method in various cancers (17-19). In OSCC, our group (15) has recently reported that the assessment of stromal TILs [as proposed by TILs Working Group (9)] can be used as a significant prognostic tool for the prediction of overall survival, disease-specific survival and disease-free survival in a large multicenter cohort of early oral tongue cancer. This assessment method has also been used successfully for other subsites of head and neck cancers (16). After further validation in large cohorts, this simple method for the assessment of TILs can be used to monitor response to immunotherapy. In addition to validation, it is important to overcome some limitations such as lack of consensus on the morphologic evaluation of TILs in OSCC and difficulty in assessing TILs using the preoperative diagnostic biopsies (20).

\section{PROGRESS OF RESEARCH ON IMMUNE BIOMARKERS OF OSCC}

In order to predict cancer response to immunotherapy, recent research (21) has tried to identify the immune profile of tumors classified into cold tumor (also known as immune desert) or hot tumor (also known as inflamed tumor). Using immunohistochemistry, several researchers have studied immune checkpoint molecules and the expression of specific TILs to identify the immune profile of OSCC. The mechanisms of such immune molecules were described in other articles $(22,23)$. Because so many studies have been published on immune biomarkers, we will focus here on the accumulated evidence from systematic reviews and meta-analyses. For example, Sievilainen et al. (24) in their recent systematic review covering the period from 1985 to 2017, on the prognostic value of immune checkpoints of OSCC have noted that seven immune checkpoints (PD-L1, FKBP51, B7-H4, B7-H6, ALHD1, IDO1, and B7-H3) had been reported to have an association with worse survival. In a meta-analysis of the prognostic value of TILs in OSCC, Huang et al. (25) found that high infiltration of CD8+ TILs, CD45RO+ TILs and CD57+ TILs associated with good survival; while high infiltration of CD163+ and CD68+ macrophages had an association with poor prognosis. In another meta-analysis, Hadler-Olsen et al. (26) found that CD163+ M2 and CD57+ had a promising relationship with outcome in patients with OSCC. Findings from these systematic reviews and meta-analyses should be considered as a cornerstone for future research in identifying the clinically most relevant immune biomarkers. It is necessary to acknowledge that the abovementioned findings were reported from studies including samples mainly from patients treated with surgery and other traditional strategies, such as radiotherapy and/or chemotherapy.

For head and neck cancer including OSCC, treatment with anti-programmed cell death-1 (anti-PD-1) and antiprogrammed cell death ligand-1 (anti-PD-L1) antibodies are crucial in the currently approved immunotherapy $(27,28)$. To 
identify which cases are more likely to benefit from such treatment, many researchers have studied the two relevant biomarkers (i.e. PD-1 and PD-L1) using samples from patients treated with immunotherapy. As an example, expression of PDL1 showed a significant association with response to durvalumab (an anti-PD-L1 antibody) in recent studies of head and neck cancer $(5,29)$. These studies found that a cutoff of $25 \%$ of cancer cells staining with PD-L1 is suitable to determine the patient's response to durvalumab immunotherapy $(5,29)$. In another study on the anticancer activity of pembrolizumab-based immunotherapy, however, Chow et al. (30) suggested to consider scoring of PD-L1 in both cancer cells and immune cells with a cutoff point of $1 \%$. Similarly, Emancipator et al. (31) reported that a "combined positive score", which evaluates the ratio of the number of PD-L1-expressing cells (including cancer cells and immune cells) to the number of all viable cancer cells multiplied by 100 , is a powerful tool in assessing the response to pembrolizumab.

In a phase 3 trial including 361 patients with recurrent HNSCC who received nivolumab, the patient survival was improved with this kind of immunotherapy (32). However, expression of PD-L1 was not that significant in the assessment of response to the treatment (32). This might highlight the difficulty in comparing the findings across the studies that have used PD-L1 as a predictive marker if the immunotherapeutic agents were different. In addition, it is important to take into consideration that the above-mentioned findings on PD-1 and/ or PD-L1 were reported from studies that included different subsites of head and neck cancer with well-known variation in their clinical behavior. Therefore, further trials should consider specific studies on OSCC to confirm the usefulness of PD-1 and PD-L1 in predicting the response to immunotherapy. In addition, whether to evaluate the expression of PD-L1 in both cancer cells and immune cells or only in immune cells needs to be determined based on the future studies. Furthermore, methods other than immunohistochemistry to assess immune biomarkers, such as immune-related signature, should be tested in OSCC cases treated with immunotherapy as this method has showed a good predictive value to immunotherapy in other tumors $(33,34)$.

\section{IMMUNOSCORE FOR OSCC}

Recent research efforts have introduced an immune-based assay known as immunoscore based on the assessment of a combination of immune biomarkers to identify the outcome of cancer (35). The most promising results with immunoscore have been reported in colorectal cancer where a scoring system for the quantification of CD3 and CD8 were standardized and showed a promising predictive power superior to TNM staging system (36) and showed successful results in phase 3 clinical trials (37). For oral carcinoma, identification of immune feature-based prognostic score has been recently introduced by Zhou et al. (38) who reported a promising prognostic value for an immunoscore based on the evaluation of CD3 in central areas and at invasive margins of OSCC; CD8, CD45RO, and FOXP3 in the central part of OSCC; FOXP3 and CD45RO at invasive margins of OSCC. However, the proposed immunoscore for OSCC will require further validation.

\section{DIGITAL PATHOLOGY AND IMMUNE BIOMARKERS}

Automated assessment of immune biomarkers has been widely studied in different cancers with successful performance (39-41). In OSCC, such assessment is still at an early stage as only few studies have reported on this concept. However, those few reports have shown promising findings. Shaban et al. (2019) reported a digital score for objective quantification of TILs that can successfully predict disease-free survival in OSCC and showed a better prognostic value than the manual assessment of TILs (42). Of note, this method of assessing TILs using wholeslide images of hematoxylin and eosin (HE)-stained sections was also successfully used in other cancers (43). In another recent study, Huang et al. (44) reported a promising value for digital image analysis of CD8 in a large cohort of tongue cancers. This approach of evaluating immune markers using digital analysis can be a simple tool to assess the immune response of OSCC and therefore validation studies are required.

\section{OTHER FACTORS TO ASSESS RESPONSE TO IMMUNOTHERAPY}

In addition to immune response and immune biomarkers, other existing factors including tumor mutational burden and mutational signatures might be associated with response to immunotherapy (45). Tumor mutational burden, referring to number of somatic mutations per coding area of a tumor genome, has shown a prognostic value in many cancers (46). Of note, recent research has showed that tumor mutational burden has a significant value in prediction of response to the immunotherapy (45). In a cohort including cases of head and neck cancer, Cristescu et al. (47) found that tumor mutational burden and $\mathrm{T}$ cell-inflamed gene expression profile can together predict the clinical responses to immunotherapy with pembrolizumab, and a longer survival was reported with higher levels of these two factors. Although pembrolizumab has been recommended for cases with high tumor mutational burden ( $\geq 10$ mutations/megabase), some researchers have caveated against such universal threshold, and highlighted the fact that patients with cancer are often receiving cytotoxic chemotherapies that might cause higher level of tumor mutational burden (48). Thus, it is still necessary to determine the optimal cutoff point for tumor mutational burden in each tumor type to identify the group that might benefit from immunotherapy. In addition, it is necessary to take into consideration that the tumor immune microenvironment is characterized by a complexity that warrants assessment of the 
clinical response from different aspects, and the measurement of tumor mutational burden being one of them.

\section{CONCLUSIONS AND PERSPECTIVES}

In the rapidly evolving field of immunotherapy, identification of biomarkers to predict the immune response can make such a therapy one of the clinically effective treatments of OSCC. There are many parameters/biomarkers and methods that have been introduced during the last three decades for the assessment of immune response. Ongoing research efforts include use of immune response in grading of OSCC, and identification of an immunoscore for OSCC. A successful clinically relevant assessment of the immune response can be considered as a cornerstone in identifying patients who will benefit from immunotherapy and also for following up the treatment response. Evidence from recent collaborative studies and/or meta-analyses highlighting the importance of evaluation of

\section{REFERENCES}

1. Almangush A, Heikkinen I, Makitie AA, Coletta RD, Laara E, Leivo I, et al. Prognostic biomarkers for oral tongue squamous cell carcinoma: a systematic review and meta-analysis. Br J Cancer (2017) 117:856-66. doi: 10.1038/ bjc. 2017.244

2. Rivera C, Oliveira AK, Costa RAP, De Rossi T, Paes Leme AF. Prognostic biomarkers in oral squamous cell carcinoma: A systematic review. Oral Oncol (2017) 72:38-47. doi: 10.1016/j.oraloncology.2017.07.003

3. Adams JL, Smothers J, Srinivasan R, Hoos A. Big opportunities for small molecules in immuno-oncology. Nat Rev Drug Discovery (2015) 14:603-22. doi: $10.1038 / \mathrm{nrd} 4596$

4. Huang PW, Chang JW. Immune checkpoint inhibitors win the 2018 Nobel Prize. BioMed J (2019) 42:299-306. doi: 10.1016/j.bj.2019.09.002

5. Zandberg DP, Algazi AP, Jimeno A, Good JS, Fayette J, Bouganim N, et al. Durvalumab for recurrent or metastatic head and neck squamous cell carcinoma: Results from a single-arm, phase II study in patients with $>/=25 \%$ tumour cell PD-L1 expression who have progressed on platinumbased chemotherapy. Eur J Cancer (2019) 107:142-52. doi: 10.1016/ j.ejca.2018.11.015

6. Schoenfeld JD, Hanna GJ, Jo VY, Rawal B, Chen YH, Catalano PS, et al. Neoadjuvant Nivolumab or Nivolumab Plus Ipilimumab in Untreated Oral Cavity Squamous Cell Carcinoma: A Phase 2 Open-Label Randomized Clinical Trial. JAMA Oncol (2020) 6:1563-70. doi: 10.1001/ jamaoncol.2020.2955

7. Maman S, Witz IP. A history of exploring cancer in context. Nat Rev Cancer (2018) 18:359-76. doi: 10.1038/s41568-018-0006-7

8. Giraldo NA, Sanchez-Salas R, Peske JD, Vano Y, Becht E, Petitprez F, et al. The clinical role of the TME in solid cancer. Br J Cancer (2019) 120:45-53. doi: 10.1038/s41416-018-0327-z

9. Salgado R, Denkert C, Demaria S, Sirtaine N, Klauschen F, Pruneri G, et al. The evaluation of tumor-infiltrating lymphocytes (TILs) in breast cancer: recommendations by an International TILs Working Group 2014. Ann Oncol (2015) 26:259-71. doi: 10.1093/annonc/mdu450

10. Fridman WH, Zitvogel L, Sautes-Fridman C, Kroemer G. The immune contexture in cancer prognosis and treatment. Nat Rev Clin Oncol (2017) 14:717-34. doi: 10.1038/nrclinonc.2017.101

11. Anneroth G, Batsakis J, Luna M. Review of the literature and a recommended system of malignancy grading in oral squamous cell carcinomas. Scand J Dent Res (1987) 95:229-49. doi: 10.1111/j.1600-0722.1987.tb01836.x
TILs and other immune biomarkers as a robust tool reveal the status of the immune response and have a strong correlation with survival outcome. There is an urgent need for validation studies to confirm the findings on these biomarkers, thus, to aid in identification of an ideal biomarker/s to select OSCC cases that can benefit from immunotherapy and to assess the patient's response. Digital assessment of immune biomarkers in OSCC are still at an early stage and require further research. Similarly, findings on the predictive value of tumor mutational burden and mutational signatures still require further research before they can be added in the personalized prediction of OSCC treatment response.

\section{AUTHOR CONTRIBUTIONS}

All authors listed have made a substantial, direct, and intellectual contribution to the work and approved it for publication.
12. Bryne M, Koppang HS, Lilleng R, Kjaerheim A. Malignancy grading of the deep invasive margins of oral squamous cell carcinomas has high prognostic value. J Pathol (1992) 166:375-81. doi: 10.1002/path.1711660409

13. Brandwein-Gensler M, Teixeira MS, Lewis CM, Lee B, Rolnitzky L, Hille JJ, et al. Oral squamous cell carcinoma: histologic risk assessment, but not margin status, is strongly predictive of local disease-free and overall survival. Am J Surg Pathol (2005) 29:167-78. doi: 10.1097/01.pas.0000149687. 90710.21

14. Bjerkli IH, Hadler-Olsen E, Nginamau ES, Laurvik H, Soland TM, Costea DE, et al. A combined histo-score based on tumor differentiation and lymphocytic infiltrate is a robust prognostic marker for mobile tongue cancer. Virchows Arch (2020) 477(6):865-72. doi: 10.1007/s00428-020-02875-9

15. Almangush A, Bello IO, Heikkinen I, Hagstrom J, Haglund C, Kowalski LP, et al. Stromal categorization in early oral tongue cancer. Virchows Arch (2020). doi: 10.1007/s00428-020-02930-5

16. Almangush A, Leivo I, Makitie AA. Overall assessment of tumor-infiltrating lymphocytes in head and neck squamous cell carcinoma: time to take notice. Acta Otolaryngol (2020) 140:246-8. doi: 10.1080/00016489.2020.1720284

17. Hwang C, Lee SJ, Lee JH, Kim KH, Suh DS, Kwon BS, et al. Stromal tumorinfiltrating lymphocytes evaluated on $\mathrm{H} \& \mathrm{E}$-stained slides are an independent prognostic factor in epithelial ovarian cancer and ovarian serous carcinoma. Oncol Lett (2019) 17:4557-65. doi: 10.3892/ol.2019.10095

18. Liu JY, Yang GF, Chen FF, Peng CW. Evaluating the prognostic significance of tumor-infiltrating lymphocytes in solid tumor: practice of a standardized method from the International Immuno-Oncology Biomarkers Working Group. Cancer Manag Res (2019) 11:6815-27. doi: 10.2147/CMAR. S201538

19. Fuchs TL, Sioson L, Sheen A, Jafari-Nejad K, Renaud CJ, Andrici J, et al. Assessment of Tumor-infiltrating Lymphocytes Using International TILs Working Group (ITWG) System Is a Strong Predictor of Overall Survival in Colorectal Carcinoma: A Study of 1034 Patients. Am J Surg Pathol (2020) 44:536-44. doi: 10.1097/PAS.0000000000001409

20. Bello IO, Wennerstrand PM, Suleymanova I, Siponen M, Qannam A, Nieminen P, et al. Biopsy quality is essential for preoperative prognostication in oral tongue cancer. APMIS (2020) 129(3):118-27. doi: 10.1111/apm.13104

21. Chen DS, Mellman I. Elements of cancer immunity and the cancer-immune set point. Nature (2017) 541:321-30. doi: 10.1038/nature21349

22. Wei SC, Duffy CR, Allison JP. Fundamental Mechanisms of Immune Checkpoint Blockade Therapy. Cancer Discovery (2018) 8:1069-86. doi: 10.1158/2159-8290.CD-18-0367 
23. Giraldo NA, Peske JD, Sautes-Fridman C, Fridman WH. Integrating histopathology, immune biomarkers, and molecular subgroups in solid cancer: the next step in precision oncology. Virchows Arch (2019) 474:46374. doi: 10.1007/s00428-018-02517-1

24. Sievilainen M, Almahmoudi R, Al-Samadi A, Salo T, Pirinen M, Almangush A. The prognostic value of immune checkpoints in oral squamous cell carcinoma. Oral Dis (2019) 25:1435-45. doi: 10.1111/odi.12991

25. Huang Z, Xie N, Liu H, Wan Y, Zhu Y, Zhang M, et al. The prognostic role of tumour-infiltrating lymphocytes in oral squamous cell carcinoma: A metaanalysis. J Oral Pathol Med (2019) 48:788-98. doi: 10.1111/jop.12927

26. Hadler-Olsen E, Wirsing AM. Tissue-infiltrating immune cells as prognostic markers in oral squamous cell carcinoma: a systematic review and metaanalysis. Br J Cancer (2019) 120:714-27. doi: 10.1038/s41416-019-0409-6

27. Cramer JD, Burtness B, Ferris RL. Immunotherapy for head and neck cancer: Recent advances and future directions. Oral Oncol (2019) 99:104460. doi: 10.1016/j.oraloncology.2019.104460

28. Moskovitz JM, Ferris RL. Tumor Immunology and Immunotherapy for Head and Neck Squamous Cell Carcinoma. J Dent Res (2018) 97:622-6. doi: 10.1177/0022034518759464

29. Rebelatto MC, Midha A, Mistry A, Sabalos C, Schechter N, Li X, et al. Development of a programmed cell death ligand-1 immunohistochemical assay validated for analysis of non-small cell lung cancer and head and neck squamous cell carcinoma. Diagn Pathol (2016) 11:95. doi: 10.1186/s13000-016-0545-8

30. Chow LQM, Haddad R, Gupta S, Mahipal A, Mehra R, Tahara M, et al. Antitumor Activity of Pembrolizumab in Biomarker-Unselected Patients With Recurrent and/or Metastatic Head and Neck Squamous Cell Carcinoma: Results From the Phase Ib KEYNOTE-012 Expansion Cohort. J Clin Oncol (2016) 34:3838-45. doi: 10.1200/JCO.2016.68.1478

31. Emancipator K, Huang L, Aurora-Garg D, Bal T, Cohen EEW, Harrington K, et al. Comparing programmed death ligand 1 scores for predicting pembrolizumab efficacy in head and neck cancer. Mod Pathol (2020) 34 (3):532-41. doi: 10.1038/s41379-020-00710-9

32. Ferris RL, Blumenschein G Jr, Fayette J, Guigay J, Colevas AD, Licitra L, et al. Nivolumab for Recurrent Squamous-Cell Carcinoma of the Head and Neck. $N$ Engl J Med (2016) 375:1856-67. doi: 10.1056/NEJMoa1602252

33. Wang Y, Chen L, Yu M, Fang Y, Qian K, Wang G, et al. Immune-related signature predicts the prognosis and immunotherapy benefit in bladder cancer. Cancer Med (2020) 9:7729-41. doi: 10.1002/cam4.3400

34. Xiao Y, Cui G, Ren X, Hao J, Zhang Y, Yang X, et al. A Novel Four-Gene Signature Associated With Immune Checkpoint for Predicting Prognosis in Lower-Grade Glioma. Front Oncol (2020) 10:605737. doi: 10.3389/fonc.2020.605737

35. Bruni D, Angell HK, Galon J. The immune contexture and Immunoscore in cancer prognosis and therapeutic efficacy. Nat Rev Cancer (2020) 20(11):66280. doi: 10.1038/s41568-020-0285-7

36. Galon J, Bruni D. Approaches to treat immune hot, altered and cold tumours with combination immunotherapies. Nat Rev Drug Discovery (2019) 18:197218. doi: 10.1038/s41573-018-0007-y

37. Pages F, Taieb J, Laurent-Puig P, Galon J. The consensus Immunoscore in phase 3 clinical trials; potential impact on patient management decisions. Oncoimmunology (2020) 9:1812221. doi: 10.1080/2162402X.2020.1812221

38. Zhou C, Diao P, Wu Y, Wei Z, Jiang L, Zhang W, et al. Development and validation of a seven-immune-feature-based prognostic score for oral squamous cell carcinoma after curative resection. Int J Cancer (2020) 146:1152-63. doi: 10.1002/ijc.32571

39. Zilenaite D, Rasmusson A, Augulis R, Besusparis J, Laurinaviciene A, Plancoulaine B, et al. Independent Prognostic Value of Intratumoral Heterogeneity and Immune Response Features by Automated Digital Immunohistochemistry Analysis in Early Hormone Receptor-Positive Breast Carcinoma. Front Oncol (2020) 10:950. doi: 10.3389/fonc.2020. 00950

40. Humphries MP, Craig SG, Kacprzyk R, Fisher NC, Bingham V, McQuaid S, et al. The adaptive immune and immune checkpoint landscape of neoadjuvant treated esophageal adenocarcinoma using digital pathology quantitation. BMC Cancer (2020) 20:500. doi: 10.1186/s12885-020-06987-y

41. Parra ER, Behrens C, Rodriguez-Canales J, Lin H, Mino B, Blando J, et al. Image Analysis-based Assessment of PD-L1 and Tumor-Associated Immune Cells Density Supports Distinct Intratumoral Microenvironment Groups in Non-small Cell Lung Carcinoma Patients. Clin Cancer Res (2016) 22:6278-89. doi: 10.1158/1078-0432.CCR-15-2443

42. Shaban M, Khurram SA, Fraz MM, Alsubaie N, Masood I, Mushtaq S, et al. A Novel Digital Score for Abundance of Tumour Infiltrating Lymphocytes Predicts Disease Free Survival in Oral Squamous Cell Carcinoma. Sci Rep (2019) 9:13341. doi: 10.1038/s41598-019-49710-z

43. Linder N, Taylor JC, Colling R, Pell R, Alvelyn E, Joseph J, et al. Deep learning for detecting tumour-infiltrating lymphocytes in testicular germ cell tumours. J Clin Pathol (2019) 72:157-64. doi: 10.1136/jclinpath-2018-205328

44. Huang Y, Lin C, Kao HK, Hung SY, Ko HJ, Huang YC, et al. Digital Image Analysis of CD8+ and CD3+ Tumor-Infiltrating Lymphocytes in Tongue Squamous Cell Carcinoma. Cancer Manag Res (2020) 12:8275-85. doi: 10.2147/CMAR.S255816

45. Oliva M, Spreafico A, Taberna M, Alemany L, Coburn B, Mesia R, et al. Immune biomarkers of response to immune-checkpoint inhibitors in head and neck squamous cell carcinoma. Ann Oncol (2019) 30:57-67. doi: 10.1093/ annonc/mdy507

46. Sha D, Jin Z, Budczies J, Kluck K, Stenzinger A, Sinicrope FA. Tumor Mutational Burden as a Predictive Biomarker in Solid Tumors. Cancer Discovery (2020) 10:1808-25. doi: 10.1158/2159-8290.CD-20-0522

47. Cristescu R, Mogg R, Ayers M, Albright A, Murphy E, Yearley J, et al. Pantumor genomic biomarkers for PD-1 checkpoint blockade-based immunotherapy. Science (2018) 362(6411):362. doi: 10.1126/science.aar3593

48. Strickler JH, Hanks BA, Khasraw M. Tumor Mutational Burden as a Predictor of Immunotherapy Response: Is More Always Better? Clin Cancer Res (2020). doi: 10.1158/1078-0432.CCR-20-3054

Conflict of Interest: The authors declare that the research was conducted in the absence of any commercial or financial relationships that could be construed as a potential conflict of interest.

Copyright (c) 2021 Almangush, Leivo and Mäkitie. This is an open-access article distributed under the terms of the Creative Commons Attribution License (CC BY). The use, distribution or reproduction in other forums is permitted, provided the original author(s) and the copyright owner(s) are credited and that the original publication in this journal is cited, in accordance with accepted academic practice. No use, distribution or reproduction is permitted which does not comply with these terms. 